$\begin{array}{ll}\text { le portiQue } & \text { Le Portique } \\ \text { Revue de philosophie et de sciences humaines }\end{array}$

$18 \mid 2006$

Heidegger. La pensée à l'heure de la mondialisation

\title{
Le cas Heidegger.
}

Un langage aux risques

\section{Henri Meschonnic}

\section{(2) OpenEdition}

Journals

Édition électronique

URL : https://journals.openedition.org/leportique/819

DOI : 10.4000/leportique.819

ISSN : $1777-5280$

Éditeur

Association "Les Amis du Portique"

Édition imprimée

Date de publication : 1 septembre 2006

ISSN : 1283-8594

Référence électronique

Henri Meschonnic, "Le cas Heidegger. », Le Portique [En ligne], 18 | 2006, mis en ligne le 15 juin 2009, consulté le 20 février 2023. URL : http://journals.openedition.org/leportique/819 ; DOI : https://doi.org/ 10.4000/leportique.819

Ce document a été généré automatiquement le 20 février 2023.

Tous droits réservés 


\title{
Le cas Heidegger.
}

\author{
Un langage aux risques
}

\author{
Henri Meschonnic
}

D'abord, quelques constatations que l'on donne pour évidentes, et qui ne le sont pas pour autant, même si elles portent sur des situations qui devraient crever les yeux et les oreilles. Il nous faut donc travailler à les rendre évidentes, pour penser à la fois la poésie et son avenir. Ce qui suppose nécessairement de réfléchir sur son passé et sur son présent.

2 Et le paradoxe, avec la poésie, mais elle n'est pas seule à être dans cet état, c'est que la poésie, comme tout ce qui est art, pensée, bref comme la vie, quand elle est humaine, a un passé, mais ce passé change selon tel ou tel regard du présent, et ce passé est pour une part qu'on ne peut pas connaître, imprévisible.

3 Mais le présent non plus n'a pas la présence qu'on lui croit. Il suffit, pour en prendre conscience, de regarder le passé. Les contemporains de toute époque sont pour la plupart les imbéciles de leur présent, du point de vue du présent qui est celui de notre regard sur ce passé qui a été leur présent. Il suffit de regarder comment Zola voyait Verlaine et Mallarmé : comme des demeurés et des déments.

4 La raison est simple. C'est que le présent, comme l'a su saint Augustin, est fait lui-même de trois présents, un présent du passé, un présent du présent, un présent de l'avenir. En ce sens, il n'y a pas plus durable que le présent, contrairement à une idée reçue, reçue chez certains philosophes surtout, que le présent n'est qu'un point sans durée aucune, puisqu'il s'enfonce à chaque instant dans le passé pour laisser venir l'avenir. Auquel cas rien n'existe, car le passé n'existe plus, l'avenir pas encore, et le présent ne cesse de passer de l'existence à l'inexistence.

5 Et pourquoi le présent de la poésie serait-il particulièrement matière d'illusion? Mais c'est parce que chez certains poètes il y a plus d'amour de la poésie que d'inventionréinvention de la poésie. Et l'amour de la poésie, dans un poème, c'est la mort du poème, la mort de la poésie. L'amour de la poésie empêche de reconnaître et même de laisser s'exprimer ce qui lui échappe, et qui est l'invention d'un sujet par un poème, corrélatif nécessaire pour qu'il y ait une invention de poème. En ce sens, les institutions culturelles du moment présent sont plus le présent du passé que le présent du présent. 
Et souvent, c'est l'avenir qui fait, après coup, sa place à ce présent. Une fois qu'il est passé. Si bien que le seul présent de ce présent est son avenir. Voilà une raison supplémentaire pour ne pas connaître ce qui est déjà inconnaissable naturellement, c'est-à-dire l'avenir de la poésie.

6 Tout cela n'est qu'une considération générale. Pertinente pour tout art, pas seulement la poésie. Pour qu'un art soit vu comme de l'art. De ce point de vue ce que nous appelons l'art des cavernes ne commence qu'en 1911, on aurait tendance à l'oublier. Tout comme l'art africain, océanien commence seulement en 1904. Et, pour revenir à la poésie, particulièrement la française, Maurice Scève commence à peine en 1918 et réellement en 1920, Jean de Sponde en 1930. Inversement, Sully Prudhomme, premier prix Nobel en 1901, n'est plus qu'un passé du passé.

7 C'est dire qu'il faut compléter l'intuition de saint Augustin, et postuler qu'il y a un passé du passé, un passé du présent, un passé de l'avenir. Tout comme un avenir du passé, un avenir du présent, un avenir de l'avenir.

8 Évidemment, il n'est pas facile de s'y retrouver. On demande des critères. Le paradoxe (à vrai dire à peine un paradoxe) avec les critères, c'est qu'ils sont nécessairement pris dans ce qu'on connaît. Et ce qu'on connaît, du moins ce qu'on croit connaître, c'est le passé. Le passé du passé et le passé du présent, qui en est l'admiration et la reproduction. Autrement dit, les critères ne servent qu'à remplacer l'aveuglement des contemporains (disons de la plupart des contemporains) sur ce à quoi le passé ne fait pas de place. En conséquence, le présent n'a de chances de commencer qu'avec la disparition de ces critères, ou plutôt et d'abord dans la lutte contre ces critères.

9 C'est ici qu'on peut, du point de vue où je me place, qui est celui de la recherche du présent et de l'avenir de la poésie, situer la pensée de la poésie chez Heidegger, et situer le rôle de la pensée de Heidegger dans la pensée et la pratique de la poésie, dans son présent et son avenir.

10 Ici intervient une première observation, indispensable et préalable à tout le reste. C'est l'unité de la pensée de Heidegger, et qui fait sa force, en même temps que sa situation. Unité de la pensée du langage, de sa pensée de la poésie, de sa pensée du politique, de sa pensée de la pensée.

11 Et la dialectique, camarades poètes, c'est que cette force fait sa faiblesse. Parce que c'est une seule et même essentialisation qui constitue, chez Heidegger, sa pensée du langage comme langue, seulement la langue, évacuation du discours (die Rede), de Sein und Zeit jusqu'à sa poétisation de la pensée de la langue et de la poésie avec les écrits sur Hölderlin des années cinquante.

12 Donc élimination du sujet du poème, l'essence de la langue étant le sujet, d'où la phrase fameuse sur l'homme qui ne parle que quand il répond à la langue. Mais cette essentialisation de la langue n'est pas séparable d'une essentialisation de la germanité comme prolongement direct de la pensée grecque, qui du coup devient elle-même en partie une abstraction. C'est de la langue allemande qu'il s'agit. Comme essence de la pensée.

13 C'est en quoi je proposais, dans Le Langage Heidegger, aux Presses Universitaires de France, en 1990 (livre insupportable à certains philosophes qui en déconseillaient la lecture, et qui observaient sur lui un pieux silence) de voir dans Heidegger un penseur expressionniste. Oui, en un sens, aussi un expressionniste allemand. Pour la part de tragique qu'il y a dans cette situation et dans cette essentialisation en chaîne de la 
pensée du langage, de la pensée de la poésie (débarrassée du poète, de ses futilités biographiques, mais aussi de la poétique même).

Et on sait bien que cette exaltation de la pensée allemande, qui rejette toutes les autres au rang de sagesses indigènes, inclut une pensée du politique qui a exalté le nationalsocialisme à une outrance telle qu'elle a été rejetée, après l'épisode du rectorat, par les nazis eux-mêmes que, il est vrai, Heidegger à son tour rejetait dans la pornographie.

C'est tout cela qui fait un bloc chez Heidegger. Et c'est ce qui est inacceptable, curieusement - mais comme on les comprend - par les heideggériens. Il n'y a que les heideggériens pour rejeter Heidegger. Eh oui, les apparences sont trompeuses.

Seuls les heideggériens ont besoin de scinder, chez Heidegger, le grand penseur et le petit nazi. Mais ils se trompent. Doublement.

Du point de vue de la pensée comme invention de la pensée, qui fait que tout ce qui n'est pas invention de pensée est du maintien de l'ordre, il y a surtout chez Heidegger un grand scolastique, un grand réaliste (au sens faussement médiéval du mot, car le réalisme n'est pas un moment passé de la pensée du langage, c'en est une donnée constitutive, immédiate, donc permanente). En ce sens, il y a une force de sa pensée, par le continu chez lui entre langage, poésie et politique, mais pas d'invention d'une pensée nouvelle du langage, d'une pensée nouvelle de la poésie, d'une pensée nouvelle du politique.

18 J'inverserais donc plutôt l'idée reçue, chez les heideggériens : un petit penseur et un grand nazi. Plus grand que les pornographes du nazisme au sens qu'il avait du sublime de la pensée allemande. Petit ne signifiant rien d'autre que le peu d'invention de problèmes nouveaux. Qui, par exemple, fusent constamment chez Wittgenstein.

Mais les heideggériens sont, historiquement, et moralement, pour le politiquement correct, obligés de séparer ce que Heidegger ne séparait pas. Ils sont donc inévitablement dans une position de faiblesse par rapport à leur grand penseur, à la fois parce qu'ils l'affaiblissent, et le trahissent, et parce que par là eux-mêmes se condamnent à être des reproducteurs d'une demi-pensée. Et quand ils sont philosophes, c'est dire qu'ils ne pensent pas. Tout en étant pénétrés du contraire. Mais ce n'est plus de la pensée, c'est du maintien de l'ordre.

Quant à ceux qui sociologisent, si je peux dire, Heidegger, en condamnant le penseur à cause de son affiliation au parti nazi (il renouvelait bien sa carte chaque année, jusqu'en 1945, on le sait), de mon point de vue, curieusement, ils sont antiheideggériens comme ils le proclament, mais leur démarche, apparemment inverse, est la même que celle des heideggériens : ils divisent ce qui faisait un chez Heidegger.

21 D'où l'invention d'une fausse question, c'est-à-dire d'une question académique, celle du silence de Heidegger après 1945. Et question qui touche à un moment sensible de la poésie, par la visite de Paul Celan à Heidegger. Autre cliché contemporain. Les deux se tenant.

22 Il est surprenant de constater à quel point les heideggériens (dont la figure de style préférée est de déclarer qu'ils ne le sont pas, heideggériens) ne savent pas lire, ne veulent pas lire, leur auteur de référence. Car il n'y a pas de silence de Heidegger. Après "Auschwitz ». Comme on résume la chose dans ce nom emblématique. Le pathétique assourdissant qui s'y est mis tient aussi à la naïveté de la visite de Celan, si exploitée, le grand penseur, le grand poète, et la cruauté d'une rencontre non-rencontre, du dialogue impossible. 

présente comme un poème la pensée du langage, du sujet, donc de l'éthique et du politique, qui mène le jeu chez son auteur. C'est le jeu majeur, le jeu, le combat du signe et du poème. 
Situation comparable à celle de la traduction : une traduction (mais là aussi il faut un peu d'exercice) ne montre pas d'abord le texte qu'elle est censée rendre, transposer, faire passer (comme aiment à dire les traducteurs, qui se voient complaisamment comme des passeurs). Une traduction montre d'abord la théorie du langage, ou disons la représentation du langage chez qui a traduit, et cette représentation du langage tient aussi à sa représentation de ce qu'est la poésie, ou la littérature. Elle montre, et elle ne sait pas qu'elle montre. Corrélatif du fait qu'elle efface, ne sait pas ce qu'elle efface, et ne sait donc pas qu'elle efface qu'elle efface, ce qui ne permet pas à qui lit la traduction de le savoir. En quoi une traduction du signe est une affaçante du poème.

Alors, l'avenir, dans tout ça? Mais c'est simple. L'avenir de la poésie ne peut dépendre que de la reconnaissance que l'essentialisation de la poésie fait la mort du poème. L'avenir de la poésie ne peut être que dans l'invention et la réinvention constante de la poésie. Contre les clichés à la mode, contre les consensus, contre sa mondanisation.

Et l'avenir est toujours incertain. Or il y a une mondialisation de la pensée Heidegger, autant chez des historiens, chez des philosophes, que chez des poètes. C'est devenu un académisme, avec ses maniérismes. Et ses effets de pouvoir.

Mais l'avenir, c'est toujours dans le présent qu'il s'invente. Ou ne s'invente pas. Toute la différence entre activité et produit, energeia et ergon, comme disait Humboldt. Et le contraire de la mondialisation heideggérienne, je dirais même l'antidote au poison, c'est certainement de comprendre, chacun pour soi, que l'universel ne se fait que dans le singulier. Pas dans la " penclubisation » de la poésie qui prodigue ses complaisances, au bénéfice d'un passé du présent. Au bon entendeur, le salut du poème.

la notion même de ce qu'est penser libre. En quoi la poésie, comme le langage, ne s'oppose pas à la vie, comme le veut encore une idée reçue, mais elle appartient constitutivement, viscéralement, à ce qui fait qu'une vie est une vie humaine.

7 En quoi l'avenir de la poésie ne concerne pas seulement ceux qu'on appelle des poètes, il nous concerne tous comme sujets. Chacun dans son avenir participe de l'avenir de la poésie. Il suffit d'être présent au présent.

\section{NOTES}

1.. C'est dans Rimbaud vivant, en 1972, dans Denkerfahrungen, Expériences de pensée. Je renvoie au Langage Heidegger, p. 166-168. 


\section{RÉSUMÉS}

« Le cas Heidegger. Un langage aux risques »

La force de la pensée de Heidegger tient à sa pensée du langage, à sa pensée du politique et à sa pensée de la pensée. Mais cette force fait aussi sa faiblesse, car elle repose sur un processus d'« essentialisation » de la langue qui est en fait celui d'une « essentialisation » de la germanité. La requête poétique de la langue aboutit paradoxalement à l'élimination du discours (Rede), à l'exclusion du sujet, à la survalorisation du passé et de l'avenir au détriment du présent et, finalement, à la mort du poème.

"The Heidegger case. A language with risks"

The strength of Heidegger's thought resides in his thought of language, in his thought of politics, in his thought of thought. But this strength is also its weakness, for it is based on a process of "essentialisation" of language which is in fact an "essentialisation" of Germanness. The poetic quest of language paradoxically leads to an elimination of discourse (Rede), to the exclusion of the subject, to the overvaluation of the past and the future against the present and, finally, to the death of the poem.

“Heidegger en Irán"

Heidegger, cuando lo interpretan los discípulos de Ahman Farid (1912-1994), quien fundó en Irán el grupo de los heideggerianos islamizantes gracias a la clave mesiánica de su gurú, al que consideran como personaje profético, llega a incarnar un mal que paraliza todo espíritu crítico y arroja a las tinieblas la tradición del siglo de las luces que tanto necesitamos hoy.

"Eine dem Risiko ausgesetzte Sprache"

Die Kraft Heideggers philosophischem Denkens liegt in seiner Arbeit über die Sprache, die Politik, und in seinem Denken über das Denken. Diese Kraft beinhaltet aber auch seine Schwäche, denn sie beruht auf einem Vorgang des „Wesentlich-werden“ der Sprache, der im Grunde zu einem „Wesentlich-werden“ der Germanität wird. So führt der dichterische Werdegang der Sprache paradoxerweise zur Ausschaltung der Rede, zum Ausschluss des Subjektes. So wird der Vergangenheit einen zu grossen Wert zugeschrieben, und auch der Zukunft, dies zum Leidwesen der Gegenwart, dies bis zum Tod der Dichtung selbst.

\section{AUTEUR}

\section{HENRI MESCHONNIC}

Henri Meschonnic est professeur Émérite de linguistique à l'Université Paris (VIII), poète, essayiste et traducteur de la Bible. Il a notamment publié Hugo, la poésie contre le maintien de l'ordre (2002), Spinoza. Poème de la pensée (2002). 